\title{
The potential relationship between neurobehavioral toxicity and visual dysfunction of BDE-209 on zebrafish larvae: A pilot study
}

\section{Bin Zhang}

Tongji University

Ting Xu

Tongji University

Daqiang Yin ( $\nabla$ yindq@tongji.edu.cn )

Tongji University https://orcid.org/0000-0001-9998-0034

\section{Sheng Wei}

Tongji University

\section{Research}

Keywords: BDE-209, Neurobehavior, Vision, Zebrafish

Posted Date: December 17th, 2019

DOI: https://doi.org/10.21203/rs.2.19026/v1

License: (c) (i) This work is licensed under a Creative Commons Attribution 4.0 International License. Read Full License

Version of Record: A version of this preprint was published at Environmental Sciences Europe on February 26th, 2020. See the published version at https://doi.org/10.1186/s12302-020-00308-7. 


\section{Abstract}

Background Although listed in the Stockholm Convention, commercial Decabromodiphenyl ether (cDecaBDE) is still being produced in many factories and used as a kind of flame retardants primarily in plastic polymers and textiles. Widespread use offered many exposure ways of its major ingredient, BDE209 , to humans and the environment. Most current studies on the health effects and toxicity of BDE-209 focused on thyroid disruption, oxidative stress, neurotoxicity and reproductive function, but seldom spread light on the relationship between neurobehavioral toxicity and visual dysfunction. Using zebrafish larvae model, we hope to uncover the potential relationship between the neurobehavioral and visual effects after exposure to BDE-209.

Results BDE-209 exposure could not induce the changes of locomotion and path angle in 5 days post fertilization (dpf) larvae, however, $5 \mu \mathrm{g} / \mathrm{L}$ BDE-209 exposure caused locomotor hyperactivity and more responsive turns at $7 \mathrm{dpf}$. The social activity of $50 \mu \mathrm{g} / \mathrm{L}$ exposure group was significantly higher than the control group at $6 \mathrm{dpf}$. Besides, 5 and $50 \mu \mathrm{g} / \mathrm{L}$ exposure caused the upregulation and downregulation of four cone opsin genes, respectively. The expression of rhodopsin gene was not influenced by both concertation exposures.

Conclusion The neurobehavioral effects induced by $5 \mu \mathrm{g} / \mathrm{L}$ BDE-209 exposure were consistent with the upregulation of four cone opsins in $7 \mathrm{dpf}$ larvae. The low concentration of BDE-209 exposure caused the hyperactivity and more responsive turns of larvae possibly contributing to the disruption on the cone opsin expressions of larvae. Our results would provide the mechanism cue of neurobehavioral toxicity after BDE-209 exposure and call for more attention on the ecotoxicology studies of BDE-209.

\section{Background}

Commercial Decabromodiphenyl ether (commercial mixture, c-decaBDE) was listed as a new member of persistent organic pollutants (POPs) under the Stockholm Convention in 2017 amendment because of its environmental persistence, high potential for bioaccumulation and biomagnification, long-distance transport capacity and adverse effects, after commercial Pentabromodiphenyl ether (c-PentaBDE) and commercial Octabromodiphenyl ether (c-OctaBDE) have been banned in 2009 amendment (http://chm.pops.int/TheConvention/ThePOPs). However, c-decaBDE is still being produced in many factories and used as a kind of flame retardants primarily in plastic polymers and textiles [1]. Widespread use and slow degradation offered many exposure ways of BDE-209, the main constituent in c-decaBDE and also one of the predominant environmental pollutants in China, to humans and the environment [2, 3]. In sediments collected from Tai Lake and Dianshan Lake, two freshwater lakes in Yangtze delta area of China, BDE-209 was the most abundant congeners of poly brominated diphenyl ethers (PBDEs) accounting for $46 \%$ and $99 \%$, respectively [4]. One study detected seven kinds of PBDE congeners in both air and dust in the TV and the refrigerator recycling workshop, finding that the workers were the most substantially exposed to BDE-209, which accounted for more than $85 \%$ of the seven PBDE congeners [5]. 
In animal-based foodstuffs BDE-209 was also recognized as the most abundant congener, comprising a proportion of at least $85 \%$ of total PBDEs [6].

Studies on the health effects and toxicity of BDE-209, the highest brominated PBDEs, was not commonly seen than other congeners. Besides limited reports of acute toxicity on invertebrates [7], the researches focused on the effects of thyroid disruption, oxidative stress, and reproductive function. Thyroid disruption of rats was observed after exposure to BDE-209, including histological abnormities in structure and ultrastructure of the thyroid gland, oxidative damages of thyroid gland, and the changes of thyroglobulin contents [8]. One study showed reproductive toxicity in peripubertal mice offspring was induced by BDE-209 during lactation exposure [9]. However, even $400 \mu \mathrm{g} / \mathrm{L}$ of BDE-209 did not show genotoxicity, while $50 \mu \mathrm{g} / \mathrm{L} \mathrm{BDE}-47$ affected the cell viability in mutant cells and BDE-99 and BDE-138 began to perform effects at $200 \mu \mathrm{g} / \mathrm{L}$ or higher [10]. Recently the neurotoxicity of BDE-209 has also been getting more attention. Sun et al. showed the learning and memory abilities of rats were damaged after BDE-209 exposure during pregnancy [11]. Similarly, another study revealed that a delayed learning in a spatial memory task and a reduction in anxiety levels were observed in young adult mice after BDE-209 exposure [12]. In a neurobehavioral study using zebrafish as the test model, the F0 growth and reproduction of zebrafish were found to be disrupted by a long-term parental exposure to low dose BDE$209(0.001 \mu \mathrm{M} \sim 1 \mu \mathrm{M})$, accompanied with the behavioral of $\mathrm{F} 1$ larvae showed slow locomotion in normal conditions, and hyperactivity under light-dark stimulus [13].

The perception of exogenous information plays a vital role in animal neurobehaviors, of which the visual system is recognized as a potential emerging target of environmental pollutants [14]. The normal visual function is the guarantee of normal neurobehavior for that early neurobehavioral dysfunction of organism was connected with the function change of visual system [15]. Zebrafish model possesses advantages in such studies [16]. Our previous study demonstrated that BDE-47 exposure impaired zebrafish vision development and further altered visually guided behaviors in larvae [17]. Chen et al. also showed that acute exposure of zebrafish larvae to DE-71 (commercial penta-BDE) caused biochemical and structural changes in the eye that lead to optokinetic and phototactic behavioral alterations [18]. The organophosphorus flame retardant triphenyl phosphate (TPhP) was also proved by the same team to have ability to disturb the ocular development of zebrafish larvae [19].

Considering the abundant environmental residues and limited toxicological data of BDE-209, the neurobehavioral effects and underlying mechanisms of BDE-209 is essential for the understanding and management of its health and ecological risks. Here using zebrafish larvae model, we demonstrated the neurobehavioral toxicity and visual dysfunction of BDE-209, finding that the neurobehavioral effects induced by low dose BDE-209 exposure were consistent with the upregulation of four cone opsins in 7 dpf larvae. Based on the potential relationship between the two effects, our results would provide the mechanism cue of neurobehavioral toxicity after BDE-209 exposure and call for more attention on the ecotoxicology studies of BDE-209.

\section{Methods And Materials}




\section{Experimental model and BDE-209 exposure}

Healthy embryos, collected from copulatory adult zebrafish (Tubingen strain) soon after light stimulation, were chosen for the following exposure from 3-5 hours post fertilization (hpf) to 7 days post fertilization (dpf). One control group and two BDE-209 (AccuStandard, USA) exposure groups were employed in the assay. The vehicle of the control group was $10 \%$ Hanks' solution with $0.1 \%$ DMSO. The nominal concentration of two BDE-209 exposure groups was $5 \mu \mathrm{g} / \mathrm{L}$ and $50 \mu \mathrm{g} / \mathrm{L}$ respectively with the same vehicle. Every group had 50 embryos in a glass petri dish with exposure solution, which was half renewed every day in the exposure duration.

\section{Neurobehavioral test protocol}

Neurobehavioral tests were performed on a ZebraBox platform (ViewPoint, France) according to our previous methods $[20,21]$, which can realize automatic tracking and high-throughput screening of larvae. Locomotion, path angle, and two-fish social activity were adopted to evaluate the neurobehavioral effects induced by BDE-209 on exposed larvae at 5, 6, and $7 \mathrm{dpf}$ respectively with light stimulus. The protocol of light stimulus included an initial 10 min of light adaption, followed by three repeated cycles with 10 min of dark period and 10 min of light period.

In the locomotion and path angle tests, larvae were transferred into a 48-well microplate from the glass petri dish at $5 \mathrm{dpf}$. Each well accommodated one larva with $1 \mathrm{~mL}$ exposure solution and each group had 16 larvae. The system can record the swimming distance of larvae as the output of locomotion test. For path angle test, three angle classes, namely straight motion $\left(-10^{\circ} \sim 0^{\circ}, 0^{\circ} \sim+10^{\circ}\right)$, average turn $\left(-10^{\circ} \sim\right.$ $\left.-90^{\circ},+10^{\circ} \sim+90^{\circ}\right)$ and responsive turn $\left(-180^{\circ} \sim-90^{\circ},+90^{\circ} \sim+180^{\circ}\right)$, were defined in this test. The system can record the frequency of different classes during the whole test.

The two-fish social activity test was performed with 6-well microplates, where two larvae were placed in each well of the plate. A valid social contact was recorded by the system when the distance of two larvae was less than $0.5 \mathrm{~cm}$. The system can record the frequency of valid contacts and the duration of each contact in the total test period.

\section{Quantitative real-time PCR (qRT-PCR) analysis}

At $7 \mathrm{dpf}$, the expression of five opsin-coding genes (zfgr1, zfblue, zfuv, zfred, and rho) after BDE-209 exposure was investigated using the qRT-PCR experiment. Trizol (Invitrogen, USA) was used to extract total RNA, and cDNA reverse transcription was performed according to the instructor of High Capacity cDNA Reverse Transcription Kits (ABI, USA). The threshold cycle values for selected genes and housekeeping $\beta$-actin were used to calculate the relative RNA amounts. The analysis was performed on a 7500 Real-Time PCR System (Applied Biosystems, USA). Fold changes of genes were defined as the ratio of RNA amounts in the treatments versus the control. More details including the sequences of primers were given in Table S1 in the Supporting Information.

\section{Statistical analysis}


Matlab R2019a (Mathworks, USA) was used to pre-process the raw data of larval behaviors from the Zebrabox system, and form a processed file for statistical analysis of different behaviors. The $2^{-\Delta \Delta t}$ method was used for the relative quatification analysis of the qRT-PCR data. The software GraphPad Prism 7 was used for statistical analysis and graph drawing. The statistical differences between the treatment groups and the control group were analysed using one-way ANOVA followed by Dunnett's test and the differences were considered significant when the $p$ value was $<0.05$. Error bars in the figures represent the standard errors of mean (SEM).

\section{Results And Discussion}

\section{Locomotion Test}

The locomotion changes of 5,6 , and $7 \mathrm{dpf}$ larvae after exposue to BDE-209 were shown in Figure 1. It could be found that locomotion during dark periods was significantly much more active than that during light periods, and locomotion at $5 \mathrm{dpf}$ was more active than that at 6 and $7 \mathrm{dpf}$. The swimming distance of two BDE-209 exposure groups at $5 \mathrm{dpf}$ and $6 \mathrm{dpf}$ had no significant differences with the control group. However, the distance of $7 \mathrm{dpf}$ larvae exposed to $5 \mu \mathrm{g} / \mathrm{L}$ exposure group was longger than the control ( $p<$ 0.05), especially in the first dark and last light periods (Figure 1c).

\section{Path Angle Test}

For the tests of path angle and social activity, the data only in the dark periods was analyzed because the locomotion during the light periods was significantly much less than the dark periods. The path angles of 5,6 , and $7 \mathrm{dpf}$ zebrafish larvae after BDE-209 exposue were counted and shown in Figure 2. The angles were divided into three classes as mentioned above. In the straight motion, larvae preferred to turn right rather than left. However, during the average and responsive turns, larvae did not show the significant preference in the orientation. Consistent with locomotion results, the path angles of $5 \mathrm{dpf}$ larvae in two BDE-209 exposure groups had no significant differences with the control group. The numbers of responsive turns of 6 and $7 \mathrm{dpf}$ larvae in $5 \mu \mathrm{g} / \mathrm{L}$ exposure group were significantly more than the control group at the last dark period (Figure 2f, 2i). Straight motion and average turns at $7 \mathrm{dpf}$ also showed the same tendency but had no significant differences.

\section{Two-fish Social Activity Test}

Two-fish social activity in the dark periods of 5, 6, and $7 \mathrm{dpf}$ larvae after BDE-209 exposure was shown in Figure 3. The duration per contact between two larvae was adopted as the indicator of analysis. At $5 \mathrm{dpf}$, social activity of the first dark period was lower than the subsequent two dark periods; however, this tendency was not observed at 6 and $7 \mathrm{dpf}$. The effects on social activity of $6 \mathrm{dpf}$ larvae were concentration-dependent at the last two dark periods, in which the activity of $50 \mu \mathrm{g} / \mathrm{L}$ exposure group was significantly higher than the control group.

\section{Gene expression test}


The functions of visual opsins which lie in the photoreceptor layer of retina, are associated with photosensitivity and circadian rhythm of animals. The gene expression of four cone opsins and rhodopsin (rho) was investigated in the present study. The four cone opsins are in charge of green, red, blue, and ultraviolet light perception (zfgr1, zfred, zfblue, and $z f u v$ ). The influence of BDE-209 exposure on the expressions of larval opsin genes was shown in Figure 4. The results showed a nonlinear concentration-dependent effect of BDE-209 exposure in the expression of four cone opsins, in which 5 $\mu \mathrm{g} / \mathrm{L} \mathrm{BDE}-209$ induced the upregulation and $50 \mu \mathrm{g} / \mathrm{L} \mathrm{BDE}-209$ induced the downregulation for all genes. However, the gene expression of rod opsin ( $r h o$ ) was not changed in both exposure groups.

\section{Discussion}

The potentials of zebrafish model in studying neurobehavioral effects of environmental pollutants have been uncovering $[22,23]$. Here the classical intermittent light stimulus to larvae was adopted to study the potential relationship between the neurobehavioral toxicity and visual dysfunction induced by BDE-209, in which larvae performed a pattern of hyperactivity during the dark period followed by quiescent state during the light period [24]. The sudden dark stimulus is a signal to zebrafish larvae, which may motivate the larval instinct of shelter seeking. The driving force behind such response is dependent on the health of the visual system. The results in locomotion showed that $5 \mu \mathrm{g} / \mathrm{L} \mathrm{BDE}-209$ exposure caused hyperactivity of larvae compared with the control at $7 \mathrm{dpf}$. Path angle means the turning direction in the moving direction of larvae [25]. The results in path angle test also revealed $5 \mu \mathrm{g} / \mathrm{L}$ BDE-209 exposure caused more responsive turns at $7 \mathrm{dpf}$. Consistently with these neurobehavioral results, $5 \mu \mathrm{g} / \mathrm{L}$ of BDE209 exposure was found to induce the upregulation of gene expression of four cone opsins at $7 \mathrm{dpf}$. Therefore, it was possible that low concentration of BDE-209 exposure induced the hyperactivity and more responsive turns by disrupting the light perception of larvae. Our previous study demonstrated BDE47 exposure significantly inhibited the expressions of zfrho, zfblue and zfgr [17], while another study showed DE-71 exposure significantly upregulated the expressions of zfrho and zfgr1, but had no effects on the expressions of zfred, zfblue, and zfuv [18]. The different PBDE congeners obviously led to the different effects on the different opsin genes.

Another interesting result is that only the exposure of low concentration BDE-209 induced the significant changes in locomotion and path angle tests at $7 \mathrm{dpf}$ other than the higher concentration exposure. However, it was reported that BDE-209 at environmental relevant doses was not an acute lethal toxicant and may just pose a low risk to marine rotifers [26]. Besides the potential influences of different tested species, the difference was possibly due to the fact that BDE-209 is easy to be bioaccumulated in sediment and organisms, which could cause a 10-fold accumulation in zebrafish larvae in unspiked sediment after 8 days [27]. Some mechanism studies indicated that the toxicity of BDE-209 was through the debromination effects to more toxic lower PBDEs congeners [28]. By far most studies used to contribute neurobehavioral changes induced by pollutants to the primary effects in thyroid function [29, 30]. The hypothalamic-pituitary-thyroid axis can be evaluated to determine thyroid endocrine disruption by BDE-209 in developing zebrafish larvae [31]. However, one study found that BDE-209 impacted expression of neurological pathways and altered behavior of zebrafish larvae, but had no explicit effects 
on thyroid function, motoneuron and neuromast development [27]. Therefore, the consistent effects of neurobehavior and gene expression in our results may offer a new insight to the mechanism of neurobehavioral effects of BDE-209. The supporting evidence is from a study using apolipoprotein $E$ (apoE2) transgenic mice model, in which high dose of BDE-209 exposure retarded the eye opening of mice without affecting other developmental features [32].

Zebrafish is a highly social species, and has the shoaling behavior promoting individuals to form tight groups [33]. Two-fish social activity offered the information of contact distance and duration between two larvae, which could be regarded as the basis of the shoaling behavior. The two-fish test actually requires the larva to sense the other larva in similar age and size, and this process is mainly dependent on vision [34]. Our results showed $50 \mu \mathrm{g} / \mathrm{L} \mathrm{BDE}-209$ exposure induced more active social activity at $6 \mathrm{dpf}$, indicating high concentration BDE-209 group may affect the social activity. One study showed the relationship between social activity and thigmotaxis, a valid index of anxiety, in which the isolated zebrafish exhibited the reduced thigmotaxis and appeared to have obvious anxiolytic effects [35]. In our study, the two-fish social activity could also offer the information of anxiety to some degree, which remains to be improved in future study.

\section{Conclusion}

In general, zebrafish larvae model was used herein to investigate the potential relationship between neurobehavioral toxicity and visual dysfunction after exposure to BDE-209. The results showed that the neurobehavioral effects of $5 \mu \mathrm{g} / \mathrm{L}$ BDE-209 exposure were consistent with the upregulation of four cone opsins at $7 \mathrm{dpf}$, providing a new mechanism cue of neurobehavioral toxicity after exposure to BDE-209 and calling for more attention on the future ecotoxicology studies of BDE-209.

\section{List Of Abbreviations}

Commercial Decabromodiphenyl ether (c-DecaBDE);

Persistent Organic Pollutants (POPs);

Commercial Pentabromodiphenyl ether (c-PentaBDE);

Commercial Octabromodiphenyl ether (c-OctaBDE);

Poly brominated diphenyl ethers (PBDEs);

Triphenyl phosphate (TPhP);

Hours post fertilization (hpf);

Days post fertilization (dpf); 
Quantitative Real-time PCR (qRT-PCR).

\section{Declarations}

\section{Ethics approval and consent to participate}

The study is in accordance with guidelines approved by the Animal Ethics Committee of Tongji University.

\section{Consent for publication}

Not applicable.

\section{Availability of data and material}

Not applicable.

\section{Competing interests}

The authors declare that they have no competing interests.

\section{Funding}

This study was funded by the International Science and Technology Cooperation Program of China (2016YFE0123700), the National Major Science and Technology Project of China (2008ZX07701001-22), and Ministry of Education-Shanghai Key Laboratory of Children's Environmental Health (CEH2018075).

\section{Authors' contributions}

$B Z$ and TX were involved in the experiments and manuscript writing. BZ and WS were involved for the data analysis. BZ, DY, and TX designed the study. TX and DY contributed to correction of the manuscript. All authors read and approved the final manuscript.

\section{Acknowledgements}

Not applicable. 


\section{Authors' details}

a State Key Laboratory of Marine Geology, Tongji University, Shanghai 200092, China

${ }^{\mathrm{b}}$ Key Laboratory of Yangtze River Water Environment, Ministry of Education, College of Environmental Science and Engineering, Tongji University, Shanghai 200092, China

c Shanghai Institute of Pollution Control and Ecological Security, Shanghai 200092, P. R. China

\section{References}

1. Zhao, Q., X. Zhao, and J. Cao, Advanced Nanomaterials for Degrading Persistent Organic Pollutants, in Advanced Nanomaterials for Pollutant Sensing and Environmental Catalysis. 2020, Elsevier. p. 249-305.

2. Zezza, D., et al., Toxicological, gene expression and histopathological evaluations of environmentally realistic concentrations of polybrominated diphenyl ethers PBDE-47, PBDE-99 and PBDE-209 on zebrafish embryos. Ecotoxicology and environmental safety, 2019. 183: p. 109566.

3. Zhi, H., et al., Decarbromodiphenyl ether (BDE-209) promotes monocyte-endothelial adhesion in cultured human aortic endothelial cells through upregulating intercellular adhesion molecule-1. Environmental research, 2019. 169: p. 62-71.

4. Du, X., et al., Polybrominated diphenyl ethers and its methoxylated analogues in biota and sediment samples from two freshwater lakes in Yangtze River delta. Environmental Earth Sciences, 2017. 76(4): p. 171.

5. Zhang, M., et al., Occupational exposure characteristics and health risk of PBDEs at different domestic e-waste recycling workshops in China. Ecotoxicology and Environmental Safety. 174: p. 532-539.

6. Wang, J., et al., Tetrabromobisphenol A, hexabromocyclododecane isomers and polybrominated diphenyl ethers in foodstuffs from Beijing, China: Contamination levels, dietary exposure and risk assessment. Science of The Total Environment, 2019. 666: p. 812-820.

7. Xiong, Q., et al., Sublethal or not? Responses of multiple biomarkers in Daphnia magna to single and joint effects of BDE-47 and BDE-209. Ecotoxicology and environmental safety, 2018. 164: p. 164-171.

8. Wang, Y., et al., A comparison of the thyroid disruption induced by decabrominated diphenyl ethers (BDE-209) and decabromodiphenyl ethane (DBDPE) in rats. Ecotoxicology and environmental safety, 2019. 174: p. 224-235.

9. Sarkar, D., D. Joshi, and S.K. Singh, Maternal BDE-209 exposure during lactation causes testicular and epididymal toxicity through increased oxidative stress in peripubertal mice offspring. Toxicology letters, 2019. 311: p. 66-79. 
10. Kyunghee, J., et al., Genotoxicity of several polybrominated diphenyl ethers (PBDEs) and hydroxylated PBDEs, and their mechanisms of toxicity. Environmental Science \& Technology, 2011. 45(11): p. 5003-8.

11. Sun, W., et al., PBDE-209 exposure damages learning and memory ability in rats potentially through increased autophagy and apoptosis in the hippocampus neuron. Environmental Toxicology \& Pharmacology, 2017. 50: p. 151-158.

12. Heredia, L., et al., Behavioral effects of oral subacute exposure to BDE-209 in young adult mice: a preliminary study. Food and chemical toxicology, 2012. 50(3-4): p. 707-712.

13. He, J., et al., Chronic zebrafish low dose decabrominated diphenyl ether (BDE-209) exposure affected parental gonad development and locomotion in F1 offspring. Ecotoxicology, 2011. 20(8): p. 18131822.

14. Chen, L., et al., Accumulation of perfluorobutane sulfonate (PFBS) and impairment of visual function in the eyes of marine medaka after a life-cycle exposure. Aquatic Toxicology, 2018. 201: p. 1-10.

15. Xu, T. and D. Yin, The unlocking neurobehavioral effects of environmental endocrine disrupting chemicals. Current Opinion in Endocrine and Metabolic Research, 2019.

16. Basnet, R.M., et al., Zebrafish Larvae as a Behavioral Model in Neuropharmacology. Biomedicines, 2019. 7(1): p. 23.

17. $\mathrm{Xu}, \mathrm{T}$. , et al., Vision, Color Vision, and Visually Guided Behavior: The Novel Toxicological Targets of 2,2',4,4'-Tetrabromodiphenyl Ether (BDE-47). Environmental Science \& Technology Letters. 4(4): p. 132-136.

18. Chen, L., et al., Acute exposure to DE-71 causes alterations in visual behavior in zebrafish larvae. Environmental Toxicology \& Chemistry, 2013. 32(6): p. 1370-1375.

19. Shi, Q., et al., Acute exposure to triphenyl phosphate (TPhP) disturbs ocular development and muscular organization in zebrafish larvae. Ecotoxicology and environmental safety, 2019. 179: $p$. 119-126.

20. Zhang, B., et al., Effects of three different embryonic exposure modes of 2, 2', 4, 4'tetrabromodiphenyl ether on the path angle and social activity of zebrafish larvae. Chemosphere, 2017. 169: p. 542-549.

21. Zhao, J., T. Xu, and D.Q. Yin, Locomotor activity changes on zebrafish larvae with different 2,2',4,4'tetrabromodiphenyl ether (PBDE-47) embryonic exposure modes. Chemosphere, 2014. 94: p. 53-61.

22. Fraz, S., et al., Paternal Exposure to Carbamazepine Impacts Zebrafish Offspring Reproduction Over Multiple Generations. Environmental Science \& Technology, 2019. 53(21): p. 12734-12743.

23. Neelkantan, N., et al., Perspectives on zebrafish models of hallucinogenic drugs and related psychotropic compounds. ACS chemical neuroscience, 2013. 4(8): p. 1137-1150.

24. Burgess, H.A. and M. Granato, Modulation of locomotor activity in larval zebrafish during light adaptation. Journal of Experimental Biology, 2007. 210(14): p. 2526-2539. 
25. Palmér, T., et al., Action sequencing in the spontaneous swimming behavior of zebrafish larvae implications for drug development. Sci Rep, 2017. 7(1): p. 3191.

26. Sha, J., et al., Effects of two polybrominated diphenyl ethers (BDE-47, BDE-209) on the swimming behavior, population growth and reproduction of the rotifer Brachionus plicatilis. Journal of Environmental Sciences, 2015. 28: p. 54-63.

27. Garcia-Reyero, N., et al., Effects of BDE-209 contaminated sediments on zebrafish development and potential implications to human health. Environment international, 2014. 63: p. 216-223.

28. Nilima, G., et al., Can biotransformation of BDE-209 in lake trout cause bioaccumulation of more toxic, lower-brominated PBDEs (BDE-47, -99) over the long term? Environment International, 2011. 37(1): p. 170-177.

29. Branchi, I., et al., Polybrominated diphenyl ethers: neurobehavioral effects following developmental exposure. Neurotoxicology, 2003. 24(3): p. 449-462.

30. Fonnum, F. and E. Mariussen, Mechanisms involved in the neurotoxic effects of environmental toxicants such as polychlorinated biphenyls and brominated flame retardants. Journal of neurochemistry, 2009. 111(6): p. 1327-1347.

31. Chen, Q., et al., Bioconcentration and metabolism of decabromodiphenyl ether (BDE-209) result in thyroid endocrine disruption in zebrafish larvae. Aquatic toxicology, 2012. 110: p. 141-148.

32. Reverte, I., J.L. Domingo, and M.T. Colomina, Neurodevelopmental effects of decabromodiphenyl ether (BDE-209) in APOE transgenic mice. Neurotoxicology and teratology, 2014. 46: p. 10-17.

33. Miller, N. and R. Gerlai, Quantification of shoaling behaviour in zebrafish (Danio rerio). Behavioural brain research, 2007. 184(2): p. 157-166.

34. Dreosti, E., et al., Development of social behavior in young zebrafish. Frontiers in neural circuits, 2015. 9: p. 39.

35. Shams, S., D. Chatterjee, and R. Gerlai, Chronic social isolation affects thigmotaxis and whole-brain serotonin levels in adult zebrafish. Behavioural brain research, 2015. 292: p. 283-287.

\section{Figures}
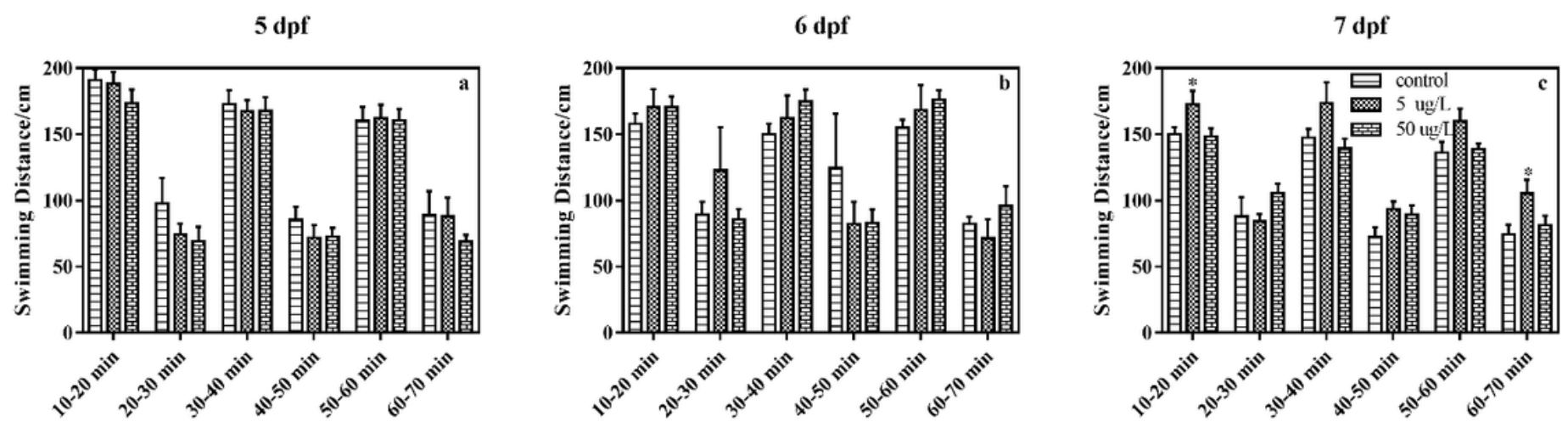
Locomotion effects of BDE-209 exposure to zebrafish larvae under dark-light stimulus (a) $5 \mathrm{dpf}$; (b) 6 dpf; (c) $7 \mathrm{dpf}$; Data was expressed as mean \pm SEM $(n=16)$. Significant difference between the control group and the exposure groups was indicated by an asterisk $\left({ }^{*} p<0.05\right)$.
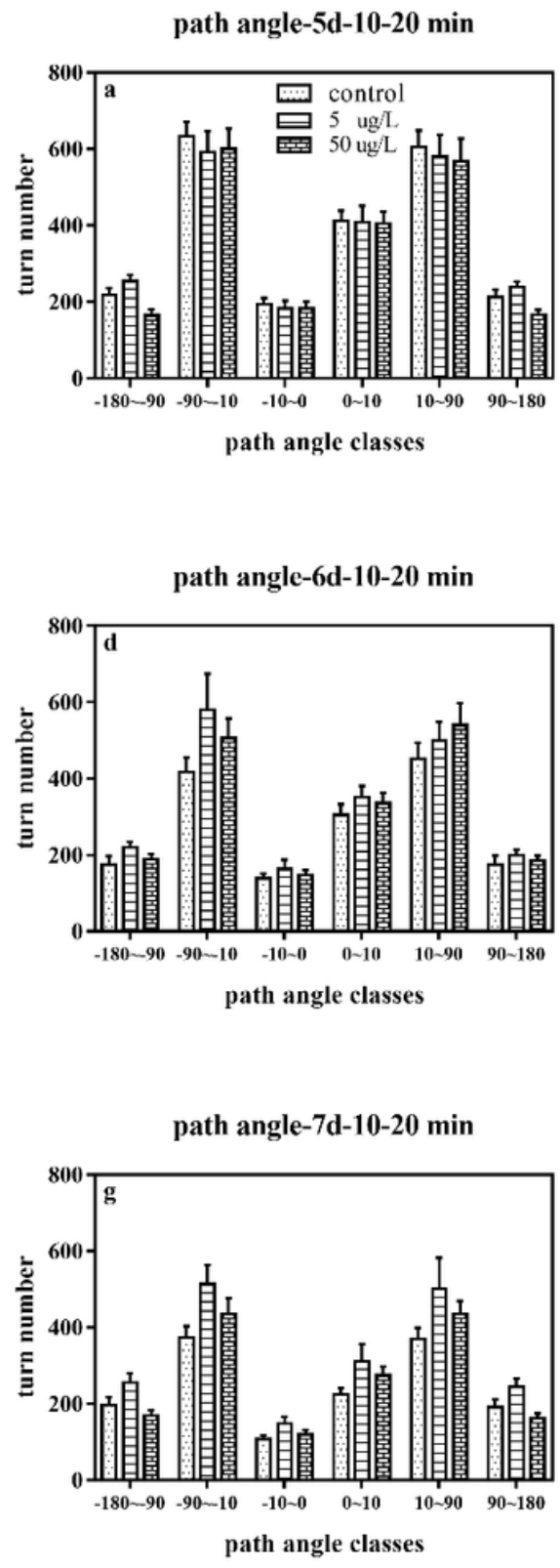

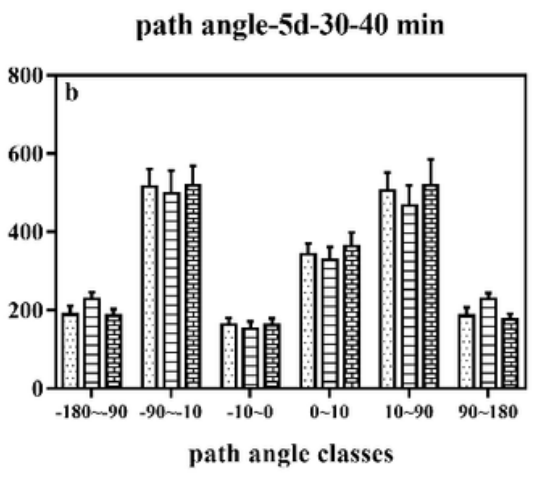

path angle-6d-30-40 min

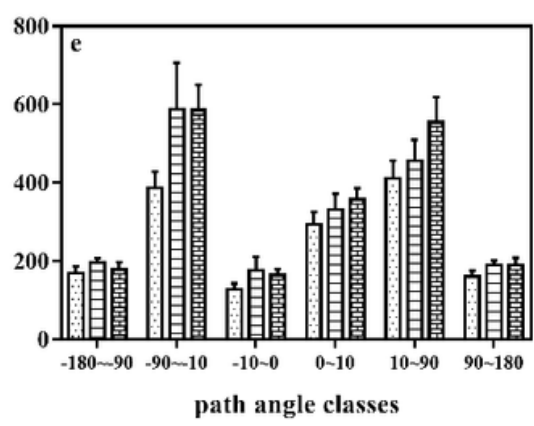

path angle-7d-30-40 min

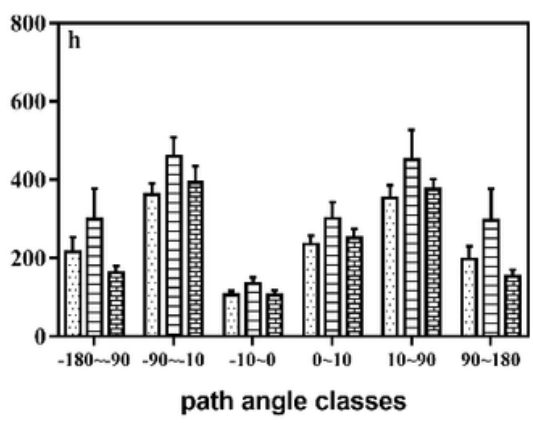

path angle-5d-50-60 min

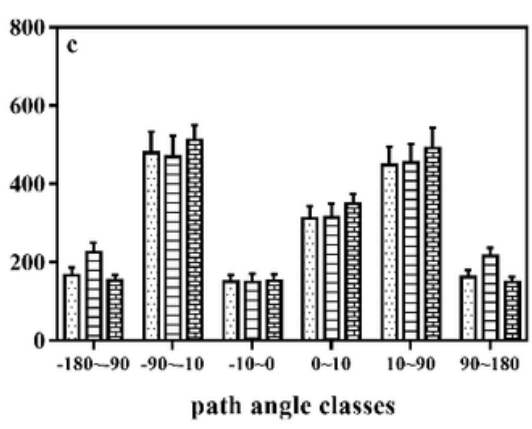

path angle-6d-50-60 min

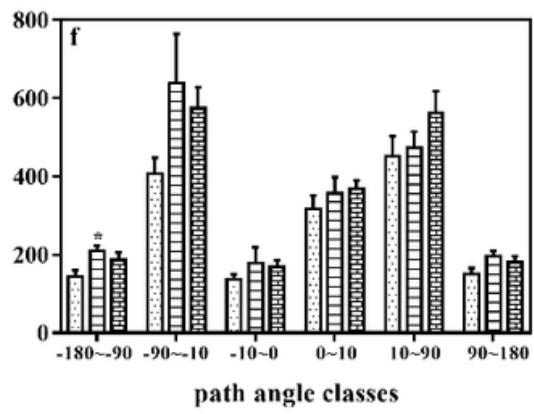

path angle-7d-50-60 min

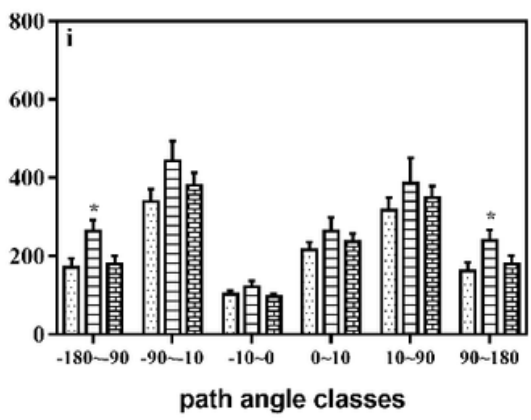

Figure 2

Path angle effects of BDE-209 exposure to zebrafish larvae during dark period (a, b, c) 5 dpf; (d, e, f) 6 dpf; $(g, h, i) 7 \mathrm{dpf}$; Data was expressed as mean \pm SEM $(n=16)$. Significant difference between the control group and the exposure groups was indicated by an asterisk $\left({ }^{*} \mathrm{p}<0.05\right)$. 
$5 \mathrm{dpf}$

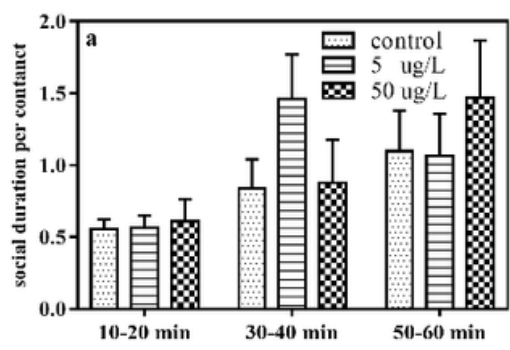

$6 \mathrm{dpf}$

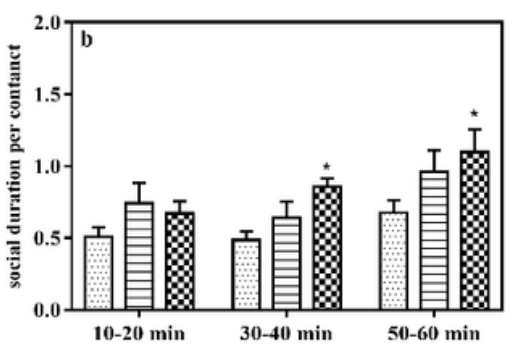

7 dpf

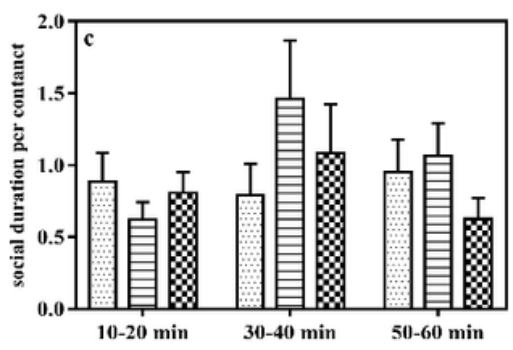

Figure 3

The effects of BDE-209 exposure on social activity of zebrafish larvae during dark period. (a) $5 \mathrm{dpf}$; (b) 6 $\mathrm{dpf}$; (c) $7 \mathrm{dpf}$; Data was expressed as mean \pm SEM $(n=6)$. Significant difference between the control group and the exposure groups was indicated by an asterisk $\left({ }^{\star} p<0.05\right)$.

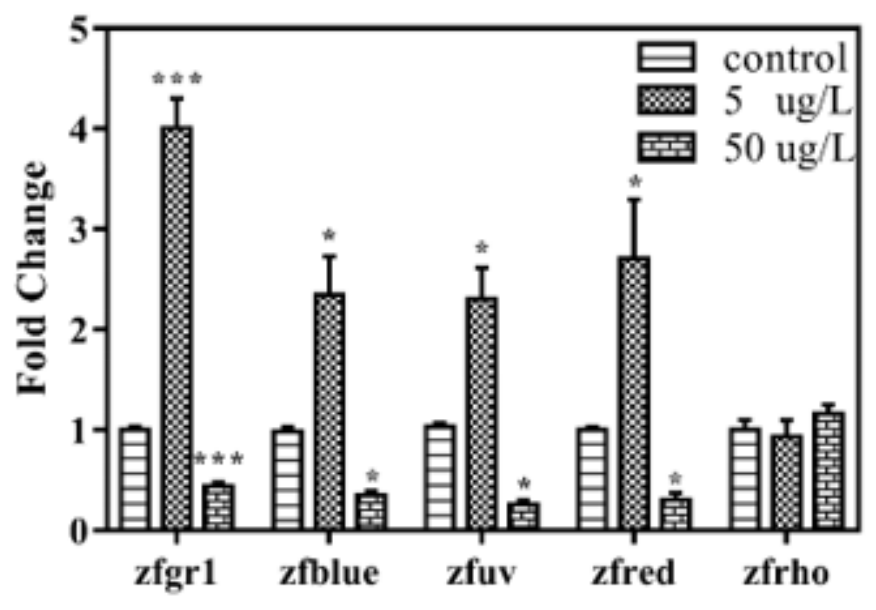

Figure 4

Gene expression effects of BDE-209 exposure to zebrafish larvae at $7 \mathrm{dpf}$ Data was expressed as mean \pm SEM $(n=3)$. Significant difference between the control group and the exposure groups was indicated by an asterisk $\left({ }^{*} p<0.05\right)$.

\section{Supplementary Files}

This is a list of supplementary files associated with this preprint. Click to download.

- SupportingInformation.docx 